\title{
Ergonomics
}

\section{Barriers to and mediators of brain-computer interface user acceptance: focus group findings}

\author{
Stefanie Blain-Moraes, Riley Schaff , Kirsten L. Gruis , Jane E. Huggins \& \\ Patricia A. Wren
}

To cite this article: Stefanie Blain-Moraes , Riley Schaff , Kirsten L. Gruis , Jane E. Huggins \& Patricia A. Wren (2012) Barriers to and mediators of brain-computer interface user acceptance: focus group findings, Ergonomics, 55:5, 516-525, DOI: 10.1080/00140139.2012.661082

To link to this article: https://doi.org/10.1080/00140139.2012.661082

册Published online: 29 Mar 2012.

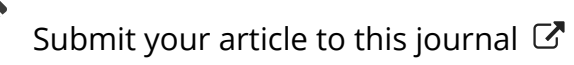

III Article views: 1075

Q View related articles $\longleftarrow$

4 Citing articles: 62 View citing articles 


\title{
Barriers to and mediators of brain-computer interface user acceptance: focus group findings
}

\author{
Stefanie Blain-Moraes ${ }^{\mathrm{a} *}$, Riley Schaff ${ }^{\mathrm{b}}$, Kirsten L. Gruis ${ }^{\mathrm{c}}$, Jane E. Huggins ${ }^{\mathrm{a}}$ and \\ Patricia A. Wren ${ }^{\text {d }}$ \\ ${ }^{a}$ Department of Physical Medicine and Rehabilitation, University of Michigan, Ann Arbor, MI, USA; ${ }^{b}$ Department of Biology, \\ University of Michigan, Ann Arbor, MI, USA; ' Department of Neurology, University of Michigan, Ann Arbor, MI, USA; \\ ${ }^{d}$ School of Health Sciences, Oakland University, Rochester, MI, USA
}

(Received 30 May 2011; final version received 19 January 2012)

\begin{abstract}
Brain-computer interfaces (BCI) are designed to enable individuals with severe motor impairments such as amyotrophic lateral sclerosis (ALS) to communicate and control their environment. A focus group was conducted with individuals with ALS $(n=8)$ and their caregivers $(n=9)$ to determine the barriers to and mediators of BCI acceptance in this population. Two key categories emerged: personal factors and relational factors. Personal factors, which included physical, physiological and psychological concerns, were less important to participants than relational factors, which included corporeal, technological and social relations with the BCI. The importance of these relational factors was analysed with respect to published literature on actor-network theory (ANT) and disability, and concepts of voicelessness and personhood. Future directions for BCI research are recommended based on the emergent focus group themes.
\end{abstract}

Practitioner Summary: This manuscript explores human factor issues involved in designing and evaluating braincomputer interface (BCI) systems for users with severe motor disabilities. Using participatory research paradigms and qualitative methods, this work draws attention to personal and relational factors that act as barriers to, or mediators of, user acceptance of this technology.

Keywords: brain-computer interface; amyotrophic lateral sclerosis; focus group; actor-network theory; qualitative research

\section{Introduction}

What am I going to do without my hands? What happens when I can't speak? Swallowing, I don't care so much about - so they feed me through a tube, so what? But my voice? My hands? They're such an essential part of me. I talk with my voice. I gesture with my hands. This is how I give to people. (Morrie Schwartz in Tuesdays with Morrie by Mitch Albom)

Morrie Schwartz was diagnosed with amyotrophic lateral sclerosis (ALS), a degenerative disease that causes progressive loss of motor function throughout the body in 5-15 per 100,000 people in the peak age range of 50-69 years (Sorenson et al. 2002). Individuals with ALS face the severe prospects of being unable to walk, eat and eventually even breathe without the help of assistive technologies; yet the opening quote illustrates that being unable to communicate and live in relationship with other people may be far more daunting. According to social interactionist theory, the existence of the human self hinges on successful interaction with others (Mead 1970, Cooley 1972); those who cannot engage in communicative interaction are, consequently, at risk of not being accorded personhood by others. While there are many discourses on the topics of personhood and humanness in the context of disability (Kitwood 1997, Siebers 2008, Speraw 2009), personhood is herein defined as 'a standing or status that is bestowed upon one human being by others, in the context of relationship and social being' (Kitwood 1997). Although several definitions of personhood emphasise an individual's agency (Quinton 1973, Post 1995), the definition we present in this article takes into account both an individual's autonomy and rational capability and that person's ability to live in relationship with others. For those who have lost their ability to engage in communicative interaction, their ability to live in relationship is compromised, making the loss of personhood a potential additional devastating consequence of conditions such as ALS.

To ameliorate this potential consequence of ALS, many augmentative and alternative communication (AAC) technologies are available that utilise residual motor movements as communication channels. In cases of extreme disability, when no reliable motor movement remains available to an individual, brain-computer interfaces (BCIs) have been proposed for communication (Wolpaw et al. 2002). By translating signals recorded directly from the brain into computer output or environmental control, BCIs may enable a motionless, voiceless individual to

\footnotetext{
*Corresponding author. Email: sblain@umich.edu
} 
articulate autonomy and subjectivity, communicate and develop relationships, thereby continuing to affirm their personhood.

To date, research efforts in BCIs have focused predominantly on improving the speed and accuracy of the technology; relatively, little attention has been paid to the human factor issues that need to be addressed before BCIs become a practical technology for potential users, such as interface design and usability. Only a small handful of studies have collected user opinions of BCIs; those that have primarily report anecdotal evidence (Sellers et al. 2010, Townsend et al. 2010) or ratings of user satisfaction (e.g. on a five-point Likert scale) (MunBinger et al. 2010, Riccio et al. 2011). The most notable effort to explore the perspectives of BCIs users is a telephone survey of the opinions and priorities of 61 individuals with ALS regarding BCI design (Huggins et al. 2011). However, the results of this study were limited by the fact that survey participants had little to no BCI interaction; hence, their answers were based on the BCI performance they imagined to be acceptable as opposed to their lived experience with a BCI. Little is yet known about this human-technology interface in individuals with severe motor disabilities and its relationship to the autonomy, communication and perception of personhood of both the users and their caregivers. For example: What are the top functional priorities that users need in a BCI? What factors influence their decision to adopt BCI technology? How do technologically complex environments and invasive technologic devices affect communication and relationships? The development of a practical BCI that users would be willing to use in their day-to-day lives hinges upon whether or not $\mathrm{BCI}$ designers and developers take the answers to such questions into account.

To address such questions, we turn to qualitative methodologies that enable us to explore the nature of an individual's experience and their transactions with themselves, others and BCI technology. Engineering methodologies and post-positivist research paradigms that have dominated the $\mathrm{BCI}$ literature to date are distinctly unsuited to address these issues (Morgan 2007). Unlike quantitative methodologies such as questionnaires and surveys (Huggins et al. 2011), qualitative methodologies enable us to describe the perspectives of individuals with ALS using their own words, rather than numerical data derived from predetermined rating systems (Portney and Watkins 2008). While quantitative methods enable researchers to determine cause and effect, qualitative methods such as focus groups enable researchers to explore how individuals perceive their own experience within a specific social context, to identify relevant issues and thus, to subsequently set research questions that are aligned with the personal and environmental contexts of the individuals involved in their research. Such research methodologies are well established in the social sciences, but the methods, assumptions, paradigms and terminology appropriate to address these issues may not be familiar to most BCI researchers. In this article, we have therefore attempted to speak to both audiences by pairing terms used in qualitative analysis paradigms with terms used in BCI research. Furthermore, the nature of these research questions invites potential users of $\mathrm{BCI}$ technology to participate in defining priorities and providing perspectives, which is well-aligned with the current emphasis on participatory research in health-related studies. Indeed, it has been shown that involving prospective users as participants in research and planning can enhance the effectiveness of interventions and save time and money in the long term (Cornwall and Jewkes 1995).

Thus, the goal of this article is to present findings on BCI user experience, priorities and opinions from a focus group of individuals with ALS and their caregivers who were familiar with BCI technology. The findings will be used to inform the future design and development of BCIs that are acceptable and appropriate for the ultimate users of this communication technology. Further, application of these methods to the BCI field may promote the adoption of such research paradigms and methodologies for future investigation of issues vital to the adoption of BCIs in the everyday life of individuals with severe motor disabilities.

\section{Methods}

Focus group participants were recruited from individuals with ALS who had previously used a P300-based BCI speller with a visual display as part of a research study. Caregivers of these individuals, who had either observed the research protocol or witnessed the setup and operation of a P300-based BCI speller immediately prior to the focus group, were also included. To provide context for the focus group discussions, the specifications of the BCI used or observed by the study participants are briefly described herein. Brain activity from the participants was obtained through the use of an electroencephalogram (EEG) cap, which was set up by placing gel under each electrode to create an electrical connection between the EEG cap and the scalp. A $6 \times 6$ grid of letters and commands was presented on a computer screen in front of the BCI user. Rows and columns of the grid flashed in a seemingly random manner while the user counted the flashes of the desired letter or command. After a specified number of flashes, the BCI selected the letter that user desired and presented it on the screen. The setup of the P300-based BCI is depicted in Figure 1.

Perspectives on BCIs were collected in a single focus group consisting of both individuals with ALS and caregivers of individuals with ALS. All participants responded independently to the focus group discussions without the use of 


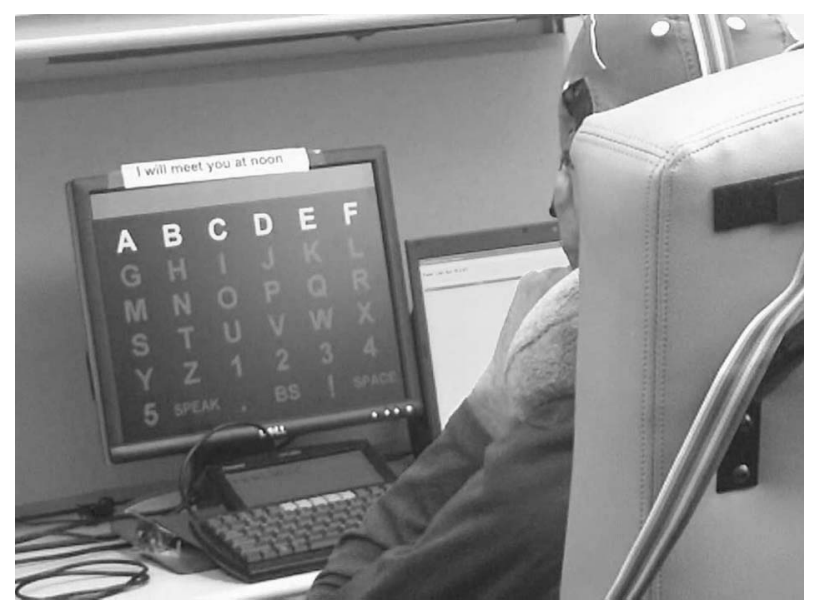

Figure 1. The setup of the P300-based BCI experienced by focus group participants with ALS. Individuals sat in front of a computer screen which displayed a $6 \times 6$ grid of letters and commands; rows and columns of the grid flashed in a seemingly random manner. Brain activity was recorded from the EEG cap donned by the BCI user.

communicative assistive technology, though caregivers occasionally needed to repeat and clarify what an individual with ALS had said, so that it was audible and comprehensible for all focus group participants. Permission to engage in research involving human subjects was obtained from the Institutional Review Board at The University of Michigan, and written informed consent was obtained from all participants prior to the start of the focus group. The focus group protocol consisted of open-ended questions probing participants' impressions of, desires for and concerns about BCIs. The focus group was transcribed in full and data were analysed for patterns, themes and narrative descriptions of participant perspectives towards BCIs using NVivo7 software (QSR International). A coding scheme was developed based on the project goals, focus group questions and themes that emerged from the focus group (Charmaz 1983). The data were explored using qualitative techniques including basic content analysis, theme identification techniques and exploration of the relations between themes and participant identities. Focus group themes will be presented in the results section of this article. Once emergent themes were identified, data analysis was guided by published theoretical and/or research literature about voicelessness and personhood (Kitwood 1997, Happ 2000) and actor-network theory (ANT) and disability (Moser and Law 1999, Moser 2006). This analysis will be presented in the discussion section of this article, followed by recommendations for future $\mathrm{BCI}$ research that may remove barriers to and augment mediators of BCI acceptance in individuals with severe motor impairments.

\section{Results}

\section{Participants}

Descriptive characteristics of the eight individuals with ALS who participated in the focus group are presented in Table 1. The revised Amyotrophic Lateral Sclerosis Functional Rating Scale (ALSFRS-R) is a validated rating instrument for monitoring the level of physical function of an individual with ALS, with a score of 48 indicating no impairment and a score of 0 indicating an individual without speech and purposeful limb movement, requiring a ventilator and feeding tube (Cedarbaum et al. 1999).

Nine caregivers participated in the focus group; descriptive characteristics of the caregivers are presented in Table 2.

Each of the participants will be referred to throughout the manuscript by their corresponding participant ID with Participant I\# referring to persons with ALS and Participant C\# indicating caregivers. Furthermore, a social unit will refer to an individual with ALS and all of his/her caregivers who participated in the study. In this focus group, there are three variations of a social unit: individual $(n=1)$, dyad $(n=5)$ and triad $(n=2)$.

\section{Emergent themes}

Unanimously, the participants' responses to BCI technology were very positive. Focus group members described the technology as offering 'freedom', 'hope' and 'connection', and described themselves when considering the technology as feeling 'impressed' and 'unlocked'. Participants described how the technology filled an important unmet need in 
their lives and highly valued the opportunities it afforded. 'It offers us opportunities that we would have no other way. Very frankly and honestly I'm thrilled about it' (C7). 'It's really regained independence, something has been taken away, and now you can get it back again' (C6b). The ensuing discussion regarding mediators and barriers towards the acceptance of a BCI for practical, day-to-day use yielded two categories: personal factors and relational factors. Each of the identified themes within these categories is fully described in the subsequent sections; a summary of the themes is provided in Table 3.

\section{Personal factors}

Personal factors were identified in the thematic analysis as being pivotal in influencing perspectives towards BCIs in both individuals with ALS and their caregivers. These factors encompass all (1) physiological, (2) psychological and (3) physical issues that mediated or blocked the acceptance of a BCI for practical, day-to-day use.

Physiological considerations involved issues of fatigue and endurance. All of the participants with ALS had experienced the BCI as part of a separate research study. While the benefits of the technology in a short-term

Table 1. Characteristics of individuals with ALS.

\begin{tabular}{|c|c|c|c|c|}
\hline Participant ID & Age & Gender & ALSFRS-R score & Experience with BCI \\
\hline I1 & 56 & M & 26 & 3 sessions \\
\hline $\mathrm{I} 2$ & 62 & $\mathrm{~F}$ & 25 & 3 sessions \\
\hline $\mathrm{I} 3$ & 58 & M & 26 & 3 sessions \\
\hline I4 & 56 & M & 18 & 3 sessions \\
\hline I5 & 62 & M & 38 & 3 sessions \\
\hline I6 & 64 & $\mathrm{~F}$ & 20 & 2 sessions \\
\hline I7 & 78 & $\mathrm{~F}$ & 37 & 3 sessions \\
\hline I8 & 78 & M & 29 & 3 sessions \\
\hline
\end{tabular}

Table 2. Characteristics of caregivers of individuals with ALS.

\begin{tabular}{lccl}
\hline Participant ID & Age & Gender & Relationship to individual with ALS \\
\hline C1 & 54 & F & Wife of I1 \\
C2 & 64 & M & Husband of I2 \\
C3 & 65 & F & Wife of I3 \\
C4a & 65 & $\mathrm{M}$ & Wife of I4 \\
C4b & 30 & F & Son of I4 \\
C6a & 64 & F & Sister-in-law of I6 \\
C6b & 64 & M & Friend of I6 \\
C7 & 73 & F & Husband of I7 \\
C8 & 48 & & Daughter of I8 \\
\hline
\end{tabular}

Table 3. Emergent focus group themes.

\begin{tabular}{llll}
\hline Theme & \multicolumn{1}{c}{ Category } & \multicolumn{1}{c}{ Issue } & $\begin{array}{c}\text { Number of associated } \\
\text { social units }\end{array}$ \\
\hline Personal factors & Physiological & Fatigue and endurance & 4 \\
& Psychological & Fatigue & 4 \\
& Psychological & Anxiety & 3 \\
& Psychological & Participant attitude toward technology & 6 \\
& Psychological & Managing distractions & 5 \\
Relational factors & Physical & Pain and discomfort & 3 \\
& Corporeal & Electrode type & 6 \\
& Technological & Relationship between BCI and other existing hardware & 5 \\
& Technological & Relationship between BCI and other existing software & 6 \\
& Social & Influence on relationship between individual with & 8 \\
& Social & ALS and caregiver & 3 \\
& Social & Training and support personnel & 5 \\
\hline
\end{tabular}


laboratory setting were clear, four of the eight social units expressed concerns regarding their ability to use the BCI for an extended period of time. 'I found that after two hours, it was really tiring, and I was done in'. (I4) Four individuals with ALS expressed concerns over physiological aspects of fatigue, such as eye strain, eye fatigue and dry eyes following an approximate half-hour use of the P300 speller.

Four of the eight social groups also expressed concern over the psychological issue of cognitive fatigue involved in using a BCI. These participants doubted their psychological capacity to manage fatigue caused by using the P300 speller, and believed that their endurance would limit the usability of this technology. '...I don't know, I just wonder what my stamina would be... I personally wonder how long I'd be able to do that... I have doubts that I'd be able to do it for, like, over an hour or so at a time'. (I5). The psychological issues of anxiety, participant attitude and managing distractions also emerged as issues of significance. Three individuals with ALS expressed concern about their psychological capacity to manage the anxiety caused by interacting with the P300 speller interface, which flashed over 300 selections each minute of use. 'I had a concern because of the lights and the flashing, my anxiety level seemed to be picking up toward the end' (I5). Current implementations of BCIs involve a significant amount of hardware and software. Participant attitude towards technology was significantly linked to their overall disposition towards BCIs. Some participants expressed confidence (I1 and C1; I3 and C3; I7 and C7; I5). Those who were comfortable with technology, expressed confidence in their ability to learn to autonomously use and operate a BCI. 'I don't really have a problem, I've used computers a lot, so I just asked my husband, and he figures I can do it, too' (C3). Others expressed grave concern (I4; C4a and C4b; I8 and C8) regarding the sophistication of the technology involved in this form of communication. Those who were uncomfortable with technology found BCIs overwhelming and were concerned about their ability to use the technology independently. '... I'm daunted by the process, and very, very concerned about my own ability to trash the whole thing.... And all of this is be beyond my capacity to do well, I feel. ... how can this be made accessible to the computer illiterate or technologically illiterate, inexperienced, my generation of people, you know, who still remember sparkplugs?' (C4a).

Participants were eager for BCI developments that would make the technology less sensitive to internal distractions (e.g. involuntary muscle twitches or trembling) and external distractions (e.g. noises). Five of the eight social units remarked that it was psychologically challenging to maintain focus on the spelling task during inevitable, yet unexpected, events. 'And you're sitting there trying to focus on the screen, and all of the sudden the ice cube maker, you know, drops ice cubes. Your train of thought gets staggered. Same thing if a dog was barking outside, or somebody... the phone rings, just any normal noise that you would hear in a house can be distracting while you're trying to focus on that' (C8).

Finally, physical issues such as pain and discomfort caused by using the BCI were raised by three of the eight social units. "All three times she said her cap, by the time she was done doing the session, she had a slight headache. So whether the cap was too tight, or if it was a bad fit ...' (C6b). 'We didn't have air conditioning turned on, and I found it uncomfortable from the standpoint of sweatiness ... and it was a little tight too' (I5).

\section{Relational factors}

While the aforementioned personal factors were of concern and considered important, far more attention in the focus group was paid to the relational factors that influence the participants' BCI design goals. Focus group participants were cognizant of the fact that the nature of the relationship between the BCI and (1) corporeal (2) technological and (3) social factors was crucial in determining whether this technology could be successfully incorporated into their day-to-day lives (Moser and Law 2001).

The corporeal relation, or the relationship between the BCI and the physical body of the participants, was discussed at length in the focus group. Six of the eight social units expressed strong opinions about the type of electrodes used to acquire signals from the brain. Five of the eight social units were clearly unsatisfied with the gelbased electrode cap used for EEG recording, calling it 'cumbersome', 'uncomfortable' and wanting something secure '... not this fiddling' (referring to the process of getting good conduction between the electrode and the scalp). They found the inconvenience of setting up and cleaning up the conductive gel distasteful. ' I would like to see it easier to use, and not the goop in your hair. I can't think of washing my hair every day' (I7). Participants were attracted to the idea of using subdermal-electrodes implanted beneath the scalp, as these electrodes may provide reduced setup time, care and daily maintenance. 'If it made it easier on the caretaker, I'd go with the implant' (I5). However, two of the eight social units firmly opposed the idea of implanted electrodes, due to perceived risk of infection, amount of care and appearance. 'Would my hair have holes in it? ... I'd rather have a hat then' (I7). These participants were not bothered by the conductive gel. One participant's electrode preference was predominantly influenced by considerations of which genre would eventually produce the highest communication accuracy through the BCI. 
'Whether it's the cap or a hat or something you put on, to me, the end result would justify whatever the means were. If I could suck up...if I utilize the same cap, and if I got more accuracy, then it, to me, would be worth it. So to me, the ends would have to justify the means' (I1).

The technological relation, or the relationship between the BCI and other existing hardware and software, was addressed by five of the eight social units. Hardware relationships involved designing interfaces between the BCI and existing electronic devices. Three of these social units wanted to have the ability to send text messages from their BCI. 'I would like to see it text. So that you could basically send a message to anyone' (I1). It was considered equally important that these relations enabled communication to be reciprocated. 'I should be able to receive messages. If I can text out, she should be able to text in' (I1). Caregivers wanted the ability to let individuals with ALS know that they were on their way, in emergency situations or otherwise. '...but that back communication is something I've missed when something's happened - when he's fallen and he's wedged between the toilet and the wall and pushing his button, but I need to get home' (C4a). Other hardware relations that garnered attention included interfacing with the telephone, television and DVR. '[She] is a talker, and it's hard. I don't know any phone out there that is adaptable for her. And we've tried several different means of, because there are moments and times-periods of time when she's home alone, also. And yes, she has Lifeline, and she can push if she needs, but she just wants to talk to her best girlfriend' (C6b). '... the other thing is the remote control and the television. And when we're watching our DVR-all the great programs that we have recorded through the week - if he could fast forward and I wouldn't have to do that, that would be a help' $(\mathrm{C} 1)$.

Software technological relations involved designing interfaces between the BCI and programmes for communication and interaction. In comparison to the current P300 spelling interface, six of the eight social units favoured interfaces that enabled them to select pre-recorded messages with a single P300-based selection to increase their communication throughput. 'I think you need to go for shortcuts to everything because we can't spell out everything we want to say... so then a series of predetermined messages that we could choose from. Maybe 10 phrases. "I'd like to see you." "I need help with this." "The toilet is backed up." "Somebody needs to empty the dishwasher." And they would only have one [picture] indicator that we had to focus on rather than spell out [character-by-character], "Somebody needs to empty the dishwasher." (I4). Participants expressed the importance of having both shortcuts and full spelling options available to them through their BCIs. 'Well, would we need both options? Sometimes we want to pour out our hearts in three letter words, and sometimes we just need to get to the bathroom. Is there no way to balance it?' (C4a). 'I sort of envision having several different screens in front of me. One screen would have the alphabet, another screen might have images of help kinds of things that I would need, another would be symbols or whatever' (I1). Options for increasing communication throughput, such as word-prediction or computer-recognised abbreviations, attracted considerable attention during the focus group. However, it was clear that the degree of precision during communication was heavily linked to personality and preference. Three social units insisted on accuracy - 'It's gotta be perfect' (C1) - while others were contented with just getting the message across - 'Close is good enough' (I8), 'If he typed out "Have a greet day", I would know he meant, "Have a great day." So, you know, you can kind of make some adjustments" (C8).

The social relation, or the relationship between the BCI and human-human interactions, had significant influence on the attitude of the focus group participants towards BCIs. The relationship between the individual with ALS and primary caregiver was of paramount importance. Within this relationship, the possibility of using a BCI in day-to-day settings was a source of tension. On one hand, BCI use offered the possibility of connection as the social unit's intra-communication ability deteriorated. 'Just the connection - the capacity to communicate is not being taken away' (C4a). On the other hand, use of the BCI requires a considerable amount of work for the caregivers, who are often saturated with the full extent of their caring responsibilities. 'I just think that it's probably time consuming initially... I can see that with all that goes into it, we might use it maybe twice a week. Because of all the time that it's going to entail' (C1). Six of the eight social units were concerned with the amount of time and work it would take specifically to set up the BCI. However, some acknowledged that once the BCI was setup, the increased independence of the individual with ALS may grant the caregiver more time for themselves. 'It can alleviate concerns that you have, it could give you, the caregiver, more time to maybe take care of things that you need done' (C1). Many social units had developed forms of interpersonal communication that were unaffected by the presence of ALS in one individual; these social units found that the type of communication that BCIs could offer did not provided any additional benefit to what they had already established. 'After 58 years of marriage, she knows what I'm going to say anyway' (I8). Others were willing to accommodate the inconvenience introduced by the BCI for the sake of social connection. 'It has been my experience that I have had to make adjustments along the way, and the more... and I always anticipate the adjustments that I had to make, because, you know, it's my life and I have to make it as... I have to give it quality. So to me, it would just be another adjustment to make. I could foresee, you know, possibly my wife or 
someone setting it up for me each morning and then undressing it in the evening, so I just see it as another adjustment that quite frankly I'd be willing to make' (I1). All members of the social unit recognised the importance of maintaining the relationships of the individual with ALS. 'And so they'll have the freedom to be able to communicate more with others being able to use this' (C1). Participants recognised that having a $\mathrm{BCI}$ might ease frustration and allow better communication between individuals. However, this ability was often balanced against the priority of minimising the caregiver's workload.

A secondary set of social relations that were considered crucial for the acceptance and practical use of BCIs were those with technical support personnel. The necessity of thorough training on the technology was emphasised. Participants preferred training to occur in a home environment where they were most comfortable. Individuals also expected to have technical support available. 'A help line - you could call and diagnose what you think the issue is and communicate to that person and hopefully they could walk you through some possible options to take you this... And if the person on that line could not solve the problem, I would expect a service rep' (I1). The absence of this relation was considered prohibitive to the in-home use of BCIs.

Finally, the importance of the relationship between the BCI and human-human interactions was illustrated through the considerable attention garnered by the psychosocial issue of appearance. The degree to which participants were willing to sacrifice the ability to independently control their appearance, and the strength of the connection between their physical appearance and their psychological self image was significantly linked to their attitudes to BCI technology. Participants were concerned with fashion and attractiveness, expressing that it was important not merely for the sake of appearance, but also because 'it goes along with the dignity' (C8). This factor was of particular concern for female individuals with ALS and female caregivers, who expressed interest in having BCI electrode caps that could be customised in colour, and for whom perceived negative effects on physical appearance (e.g. 'Would my hair have holes in it?' [I7]) were linked to aversion towards using the technology. Conversely, some participants felt that sacrificing their appearance was a small price to pay for the ability to communicate. 'I think it would be a small price to pay for all of us to go like [him (pointing to a bald individual)] and ... be completely shaven in order to have electrodes attached to the head even if we have to shave the head completely. Once a week. And the women can wear wigs' (I4). This statement was a point of contention in the focus group, and the ensuing spirited discussion illustrated the heterogeneity of opinions, values and principles among target $\mathrm{BCI}$ users.

\section{Discussion}

The first purpose of this study was to determine the attitudes of potential BCI users towards BCI technology and to elucidate the factors that influenced them, so as to inform design goals and challenges for future BCI research. Overall, individuals with ALS and their caregivers were very enthusiastic about the prospect of using BCI technology. Focus group participants maintained that BCIs had the potential to give them freedom from the confining effects of their condition, enable them to stay connected to their loved ones and allow them to remain independent as their condition progressed. 'The person who would be able to use it could have some level of freedom now that possibly they may have lost' $(\mathrm{C} 1)$. However, participants also expressed that BCI technology in its current form would not be acceptable or appropriate for day-to-day use in practical, real-world situations. Participants identified a number of psychological, physiological and physical personal issues that could present as unyielding barriers towards the successful, real-world use of BCIs. For example, if individuals are unable to manage the physiological and psychological fatigue associated with BCI use, it is unlikely that this technology will be successfully integrated into their day-to-day communication and activity patterns. Developments in BCI technology that remove these immediate barriers towards an individual's ability to use the BCI are clearly necessary; however, the results of the focus group indicate that addressing these personal factors alone is not sufficient for successful adoption of BCI technology. Indeed, issues of corporeal, technological and social relations that influenced the integration of the BCI into the networks of the lives of each social unit garnered considerably more attention from focus group participants than the personal factors related to BCI acceptance.

\section{Theoretical understanding of the importance of relational factors}

To understand the strong emphasis that the focus group participants placed on these relational factors, we turn to ANT to understand how action is made possible for an individual with neuromuscular disabilities. Akrich (1992) and Latour (1993) suggest that actions are distributed across a network consisting of an individual, nonhuman aids and social relations to other individuals. Further, these actions are emergent effects of the heterogeneous parts that make it 
possible. Consequently, abilities and disabilities emerge from the overall successfulness of these networks of heterogenous, material and specific entities, which enable action for the individuals included in them (Moser and Law 1999, Moser 2006). (Dis)abilities result from the relations individuals have with other entities. Thus, BCIs have the potential to give ability to individuals only if they are seamlessly incorporated into the existing networks of the individuals' lives; if they are not, they will be of little practical benefit. This is consistent with the dominant themes that emerged from this focus group, which illustrate the critical need to focus upon the relationship between BCIs and these networks before they can transition from laboratory technologies to technologies that enable day-to-day action for an individual.

The psychosocial significance of voice (Happ 2000) was also underscored in the perspectives of the focus group participants. The importance of maintaining one's voice was stressed unanimously. 'My ALS began as PLS, and the first effect was my palate and my tongue. And I don't want to lose the ability to speak' (I4). '. . they don't want to lose their voice. They want to keep their voice' (C6b). AAC technologies, including BCIs, were perceived as tools that enabled an individual to express themselves by prosthetic means. 'I just think it is wonderful that you can give someone a voice who is losing theirs' (C8). However, while this idea of BCIs as a voice for individuals who are losing theirs is powerful, it is important not to adopt it as the sole philosophy for BCI design, as it ignores the complex relationship identified above wherein BCIs also enable action for individuals. The ability to maintain autonomy and independence via actions such as controlling the environment and changing the television channel, which were emphasised by focus group participants as important for maintaining a sense of agency, are not addressed through the terminology 'giving a voice'. In agreement with work done by Moser and Law (2001), we recommend recasting this terminology and instead speaking of 'articulation'. 'Our posthumanist suggestion, and it is hardly novel, is that "voices" do not exist in and of themselves. They do not reflect something that is pre-given. Rather they are constituted or "articulated" into being in material arrangements, which include social, technological and corporeal relations' (Moser and Law 2001). To speak of giving 'voices' risks limiting articulation to those which are verbal, textual or linguistic, and to ignore and disqualify 'voices' that are non-verbal. Prospective BCI users expressed interest in using the BCI to articulate autonomy, agency and subjectivity. This language supports a perspective of personhood that accounts for both an individual's autonomy and that person's ability to live in relationship with others (Kitwood 1997). It emphasises the cardinal importance of the relational aspects of an individual's self, situating BCIs outside of their traditional place as technologies that compensate for an individual's physical impairments, and instead situating them as parts of a network designed to keep an individual's personhood unimpaired. BCIs designed with this broader philosophy have powerful potential to become accepted and fully integrated into the lives of individuals with ALS and other severe neuromuscular disabilities.

\section{Future directions for BCI research}

As BCIs approach performance standards that render them reliable and accurate laboratory technologies, prospective BCI users in our focus group emphasised two complementary foci for future research towards the ultimate goal of developing a practical, in-home BCI technology. The first focus is to develop techniques to remove the barriers presented by personal factors (e.g. psychological, physiological and physical) that negatively influence the attitudes of users and their caregivers towards BCIs. A great deal of this work can be accomplished through thoughtful (re)design of BCI technology. As examples of potential future directions: psychological issues related to being daunted by the technology can be addressed through user-interface design and software development towards greater accessibility for the average computer user. Hardware solutions (e.g. microphones that detect startling environmental noises) and software solutions (e.g. signal processing and pattern classification techniques) can be developed that ameliorate the physiological effect of environmental distraction. A diversity of BCI interface options that include both gel-based electrode caps, subdermal implanted electrodes and dry electrodes should be developed to accommodate the wide range of preferences and values regarding physical appearance of prospective BCI users.

The second focus involves addressing the relational factors between BCIs and the corporeal, technological and social networks of future users. It has been shown that even if a technology leads to improved functioning, this alone will not ensure that an assistive technology will be used by the individuals in the target population (Scherer and McKee 1990, Phillips and Zhao 1993, Scherer 1993). Non-use, or abandonment of assistive technologies, occurs when users are not involved in the development, selection and integration of these technologies into their lives (Phillips and Zhao 1993). To have positive mediating effect on user acceptance of BCIs, the inconvenience of acquiring signals from the brain needs to be minimised, interfaces between BCIs and existing technologies need to be provided and the burden of this technology on the caregiver must be minimised. These types of developments must be addressed in concert with the 
personal issues and issues of classifier performance and accuracy before BCIs can become practical technologies for individuals with severe motor disabilities.

Finally, as BCIs transition from laboratory settings into the personal and environmental contexts of individuals with neuromuscular disabilities, new research methodologies are needed to (1) understand the priorities and perspectives of BCI users and (2) measure the performance and impact of BCIs within these settings. Qualitative methods (such as focus groups, as presented herein) and participatory research paradigms need to be used in parallel with more traditional reductionist and deterministic approaches in order to develop a technology that maximises the quality of life of individuals with conditions such as ALS.

\section{Limitations}

This focus group is one of the first studies to explore the perspectives and lived experiences of individuals with ALS with respect to BCI technology. While it provides valuable insights for future directions for BCI research, it is important to interpret the results within the limitations of the study. While each of the individuals with ALS had experience using a BCI, their experiences were constrained to two or three sessions in the context of a controlled experimental protocol. Thus, while grounded in experiences of other complex, frequently used technologies, the perspective of the participants on mediators and barriers to long-term, day-to-day BCI use are projected, as opposed to firsthand, experiences. Many of the personal factors that arose as barriers to $\mathrm{BCI}$ acceptance (e.g. eye fatigue, anxiety due to flashing) were specific to the participants' experience with the row-column P300-based BCI. Townsend et al. (2010) have demonstrated that users report less visual fatigue when they use a variation of the P300-based BCI with different patterns of stimulus presentation. Moreover, many themes that emerged as personal factors in this focus group do not translate to BCIs which respond to spontaneous control signals generated by the user (e.g. sensorimotor rhythm (SMR)-based BCIs). Finally, all of the individuals with ALS who participated in the focus group still had the ability to speak and to be understood by their caregivers, and thus, an established, functional means of maintaining their personhood. As a result, the themes that emerged in this focus group may not translate to individuals who do not have any other means of functional communication available to them, for whom BCI technologies are the most relevant.

\section{Conclusions}

Individuals with ALS are at risk of not being accorded personhood by others as they gradually lose their ability to communicate. This loss of communication and the resulting inability to live in relationship with other people may be one of the more devastating consequences of their condition. Overall, individuals with ALS and their caregivers were enthusiastic about using BCI technology to counteract these effects of ALS. Focus group analysis yielded two categories of key mediators of, and barriers to, user acceptance of this technology: personal factors and relational factors. Personal factors, which included physical, physiological and psychological concerns, were less important to participants than relational factors, which included corporeal, technological and social relations with the BCI. The importance of these relational factors can be understood in the context of ANT, wherein ability emerges only when technologies such as BCIs are seamlessly incorporated into the existing networks of an individual's lives. The perspectives garnered from this focus group suggest that in addition to developing BCIs with the best speed and accuracy, BCI designers should place equal priority on developing BCIs that give voice and enable action, allowing individuals to express autonomy, engage in meaningful communicative interaction and articulate their personhood. As BCIs make this transition, qualitative methods and participatory research paradigms such as those introduced in this manuscript will be needed to fully understand the broader potential effects of this technology on the lives of BCI users and their caregivers.

\section{References}

Akrich, M., 1992. The de-scription of technical objects. In: W. Bijker and J. Law, eds. Shaping technology, building society: studies in sociotechnical changes. Cambridge, MA: MIT Press, 205-224.

Cedarbaum, J.M., Stambler, N., Malta, E., Fuller, C., Thurmond, B., and Nakanishi, A., 1999. The ALSFRS-R: a revised ALS functional rating scale that incorporates assessments of respiratory function. BDNF ALS Study Group (Phase III). Journal of the Neurological Sciences, 169 (1-2), 13-21.

Charmaz, K., 1983. The grounded theory method: an implication and interpretation. In: R.M. Emerson, ed. Contemporary field research. Prospect Heights, IL: Waveland, 109-126.

Cooley, C.H., 1972. The looking glass self. In: J. Manis and B. Meltzer, eds. Symbolic interaction: a reader in social psychology. Boston, MA: Allyn \& Bacon, Inc., 231-233. 
Cornwall, A. and Jewkes, R., 1995. What is participatory research? Social Science \& Medicine, 41 (12), $1667-1676$.

Happ, M.B., 2000. Interpretation of nonvocal behavior and the meaning of voicelessness in critical care. Social Science \& Medicine, 50 (9), 1247-1255.

Huggins, J.E., Gruis, K.L., and Wren, P.A., 2011. What do brain-computer interface users want? Opinions and priorities of potential users with amyotrophic lateral sclerosis. Amyotrophic Lateral Sclerosis, 12 (5), 318-324.

Kitwood, T., 1997. Dementia reconsidered: the person comes first. Buckingham: Open University Press.

Latour, B., 1993. La clef de Berlin et autres leçons d'un amateur des sciences. Paris: La Découverte.

Mead, G.H., 1970. Development of the self through play and games. In: G.P. Stone and H.A. Farberman, eds. Social psychology through symbolic interaction. London: Ginn-Blaisdell, 537-545.

Morgan, D.L., 2007. Paradigms lost and pragmatism regained: methodological implications of combining qualitative and quantitative methods. Journal of Mixed Methods Research, 1 (1), 48-76.

Moser, I., 2006. Disability and the promises of technology: technology, subjectivity and embodiment within an order of the normal. Information, Communication \& Society, 9 (3), 373-395.

Moser, I. and Law, J., 1999. Good passages, bad passages. In: J. Law and J. Hassard, eds. Actor network theory and after. Oxford: Blackwell, 196-219.

Moser, I. and Law, J., 2003. "Making voices": new media technologies, disabilities, and articulation. Cambridge, MA: MIT Press, 491-520.

Munbinger, J.I., Halder, S., Kleih, S. C., Furdea, A., Raco, V., Hosle, A., and Kubler, A., 2010. Brain painting: first evaluation of a new brain-computer interface application with ALS-patients and healthy volunteers. Frontiers in Neuroscience, 4 (182), 1-11.

Phillips, B. and Zhao, H., 1993. Predictors of assistive technology abandonment. Assistive Technology, 5, 36-45.

Portney, L.G. and Watkins, M.P., 2008. Descriptive research. In: M. Cohen, ed. Foundations of clinical research: applications to practice. 3rd ed. London: Prentice Hall, 301-324.

Post, S.G., 1995. The moral challenge of Alzheimer disease. 1st ed. Baltimore: Johns Hopkins University Press.

Quinton, A., 1973. The nature of things. London: Routledge.

Riccio, A., Leotta, F., Bianchi, L., Aloise, F., Zickler, C., Hoogerwerf, E.J., Kubler, A., Mattia, D., and Cincotti, F., 2011. Workload measurement in a communication application operated through a P300-based brain-computer interface. Journal of Neural Engineering, 8 (2), 025028.

Scherer, M.J., 1993. Living in the state of stuck: how technology impacts the lives of people with disabilities. Cambridge, MA: Brookline Books.

Scherer, M.J. and Mckee, B., 1990. High-tech communication devices: what separates users from non-user? Augmentative \& Alternative Communication, 6, 99.

Sellers, E.W., Vaughan, T.M., and Wolpaw, J.R., 2010. A brain-computer interface for long-term independent home use. Amyotrophic Lateral Sclerosis, 11 (5), 449-455.

Siebers, T., 2008. Disability theory. Ann Arbor, MI: The University of Michigan Press.

Sorenson, E., Stalker, A.P., and Kurland, L.T., 2002. Amyotrophic lateral sclerosis in Olmsted County, Minnesota, 1925 to 1998. Neurology, 59, 280-282.

Speraw, S., 2009. 'Talk to me-I'm human': the story of a girl, her personhood, and the failures of health care. Qualitative Health Research, 19 (6), 732-743.

Townsend, G., LaPallo, B., Boulay, C., Krusienski, D., Frye, G., Hauser, C., Schwartz, N., Vaughan, T.M., Wolpaw, J.R., and Sellers, E.W., 2010. A novel P300-based brain-computer interface stimulus presentation paradigm: moving beyond rows and columns. Clinical Neurophysiology, 121 (7), 1109-1120.

Wolpaw, J.R., Birbaumer, N., McFarland, D.J., Pfurtscheller, G., and Vaughan, T.M., 2002. Brain-computer interfaces for communication and control. Clinical Neurophysiology, 113 (6), 767-791. 